\title{
Design and experiment of vertical pneumatic fertilization system with spiral Geneva mechanism
}

\author{
Haibo Chen ${ }^{1}$, Jingfu Zheng ${ }^{2}$, Shaojie Lu$^{2}$, Shan Zeng ${ }^{2 *}$, Silong Wei ${ }^{2}$ \\ (1. Experimental Basis and Practical Training Center, South China Agricultural University, Guangzhou 510642, China; \\ 2. College of Engineering, South China Agricultural University, Guangzhou 510642, China)
}

\begin{abstract}
In the traditional fertilization method, a large amount of fertilizer is applied, which causes congestion easily. It is not conducive to the sustainable development of agriculture. In this work, a vertical pneumatic fertilization system with a spiral Geneva mechanism was designed according to the operational requirements of variable-rate and smooth fertilization. The uniformity in discharging fertilizer with different spiral angles was simulated and analyzed by extended discrete element method (EDEM) based simulation software, from where the spiral angle of the fertilizer discharge wheel was determined to be $45^{\circ}$. The fertilization system includes a fertilizer apparatus with a spiral Geneva mechanism, whose optimal performance parameters were obtained from the bench experiment. The accuracy and uniformity of the fertilizer application system were taken as the evaluation indicators. The linear relationships of the discharged amount of fertilizer with the rotational speed and the opening of the fertilizer discharging wheel were verified by both static blowing fertilization and field experiments. The static blowing experimental results show that the discharged amount of fertilizer has a high linear correlation and accuracy with both the opening and rotational speed, and the highest correlation occurs with the rotational speed. The rotational speed and opening have significant effects on the discharged amount of fertilizer and the average coefficient of variation. The effect of the rotational speed was the most significant. According to the model obtained by multiple regression fittings, the optimal parameters were determined when the average coefficient of variation was small, the rotational speed was $15.9 \mathrm{r} / \mathrm{min}$ and the opening was $34.4 \mathrm{~mm}$. Therefore, the purpose of precise fertilization can be achieved by adjusting the opening or rotational speed in a way to get the exact amount of fertilizer discharged as required by the corresponding crops. The field experiment showed that the variation coefficient of each fertilizer discharged decreases first and then increases with an increasing opening under different rotational speeds, which is consistent with the theoretical value. When the opening was $40 \mathrm{~mm}$ or $50 \mathrm{~mm}$, the variation coefficient reached the minimum value, which is far less than the qualified index of $7.8 \%$. In the static blowing experiment and the dynamic field experiment, the stability of the fertilizer discharging device can be significantly enhanced with the utilization of the pneumatic conveying fertilizer. This study can provide a theoretical reference for parameter selection and optimization of vertical spiral fertilization systems.
\end{abstract}

Keywords: precision fertilization, fertilizer apparatus, variable rate technology, spiral Geneva mechanism, simulation analysis, performance experiment

DOI: $10.25165 /$ j.ijabe.20211404.6575

Citation: Chen H B, Zheng J F, Lu S J, Zeng S, Wei S L. Design and experiment of vertical pneumatic fertilization system with spiral Geneva mechanism. Int J Agric \& Biol Eng, 2021; 14(4): 135-144.

\section{Introduction}

China is one of the largest rice producers and consumers in the world $^{[1]}$. At present, fertilization is the fastest and most effective way to increase rice yield ${ }^{[2,3]}$. Nevertheless, the traditional fertilization technique not only causes a series of environmental problems but also affects the yield and the utilization efficiency of fertilizer, which are not conducive to the sustainable development of agriculture ${ }^{[4-7]}$. Variable precision fertilization can substantially improve the utilization efficiency of fertilizer, reduce

\section{Received date: 2021-03-08 Accepted date: 2021-07-05}

Biographies: Haibo Chen, $\mathrm{PhD}$, Associate Professor, research interest: agricultural mechanization, automation and information, Email: huanongchb@ scau.edu.cn; Jingfu Zheng, Undergraduate student, research interest: agricultural mechanization and automation, Email: 1599158198@qq.com; Shaojie Lu, Undergraduate student, research interest: agricultural mechanization and automation, Email: 920660104@qq.com; Silong Wei, Graduate student, research interest: agricultural mechanization and automation, Email: 2622675850@qq.com *Corresponding Author: Shan Zeng, PhD, Associate Professor, research interest: agricultural mechanization and automation, College of Engineering, South China Agricultural University, Guangzhou 510642, China. Tel: +86-13798175915, Email: shanzeng@scau.edu.cn. the amount of fertilizer requirement, and control the production cost. It can also reduce environmental pollution and directly bring economic benefits. Moreover, the application of the precise amount of fertilizer has a direct impact on crop yield and fertilizer utilization ${ }^{[8]}$.

In recent years, scholars have been studying the variable rate and precision fertilization technology, which has been applied almost at all levels ${ }^{[9-13]}$. At present, there are three main types of variable fertilization techniques, namely disk, spiral, and Geneva mechanisms ${ }^{[14-16]}$. The fertilization system with the Geneva mechanism has the advantages of simple structure, stable operation, quick response of actuator, flexible variable control. It performs double bi-variable control, which is widely used in the field of variable-rate fertilization. Zeng et al. ${ }^{[17]}$ verified by performing a control experiment that the driving layer was the key factor that affected the accuracy of fertilization. The control mechanism for opening the fertilizer discharging wheel was realized mainly in two ways: axial movement, and baffle opening and closing ${ }^{[18]}$. A two-stage spiral device for discharging fertilizer with low requirement for fertilizer forms, a 2BFJ-6 type variable rate fertilizer apparatus with outer Geneva mechanism and a precision 
variable rate fertilizer apparatus for rice and wheat were developed by Chen et al. ${ }^{[19]}$, Pan et al. ${ }^{[20]}$, and Shi et al. ${ }^{[21]}$, respectively. Some researches working on the variable fertilizer system with the Geneva mechanism have also been reported. However, the quantitative relationship between the rotational speed and the opening of the fertilizer wheel and the amount of fertilizer is still in the initial stage. Yuan et al. ${ }^{[22]}$ showed that the fertilizer discharge in the variable rate fertilization increased nonlinearly with increasing rotational speed and opening. Making a comparison between a bench test and EDEM in the study of a fertilizer discharger with Geneva mechanism, Lyu et al. ${ }^{[23]}$ established a linear relationship between fertilizer discharge and rotation speed, as well as fertilizer discharge and opening. However, their correlation degree was not high. Using a real-time embedded system as the core controller in a laboratory test, Ishola et al. ${ }^{[24]}$ found a linear relationship between the rotational speed of the fertilizer wheel and the amount of fertilizer, which was greater than 0.998. Conducting an EDEM simulation test by considering that there is no strict linear correlation between the amount of fertilizer discharge and the opening of the fertilizer discharge wheel, Zhang et al. ${ }^{[25]}$ found that the square of the linear correlation coefficient between them was 0.98. Simulating in the EDEM software, Ding et al. ${ }^{[26]}$ observed that the square of the linear correlation coefficient between the amount of fertilizer discharge and the opening of the fertilizer discharge wheel was $R^{2}=0.99$. Li et al. ${ }^{[27]}$ developed a double variable fertilizer applicator with rotational speed and opening, and, found in a bench test that there was no linear relationship between the amount of fertilizer discharge and rotational speed of the fertilizer discharge wheel. In view of the present research status, the quantitative relationship between the double-control variables of the rotational speed and opening of the fertilizer wheel and fertilizer discharge should be studied further. Moreover, the rice hole spacing is usually kept at 0.1-0.3 m, and the field operation speed is kept at $1-3 \mathrm{~m} / \mathrm{s}$, which increases the requirements for improving the uniformity and controlling of the fluctuation of fertilizer discharge ${ }^{[28]}$.

In view of the above problems, in this work, a vertical spiral fertilization system was developed and optimized aiming at the accuracy and uniformity of fertilization. The optimal spiral angle is determined by the EDEM software. The effects of the rotational speed and opening of the fertilizer discharging wheel on the fertilizer discharging conditions were studied through static blowing experiments as well as field experiments, which provide a theoretical reference for parameter selection and optimization of vertical spiral fertilization systems.

\section{Structure and working principle of vertical fertilization system with spiral Geneva mechanism}

\subsection{Overall design of vertical fertilization system with spiral Geneva mechanism}

The vertical fertilization system with spiral Geneva mechanism is installed by a $2 \mathrm{BDH}-10$ type precision rice hill-drop drilling machine for dry land. As shown in Figure 1, it is composed of a rotational speed control motor, rotary shaft, opening control motor, opening control mechanism, vertical fertilizer apparatus with spiral Geneva mechanism, fertilizer bin, fan, and pneumatic conveying pipes. In order to perform bi-variable control of the rotational speed and wheel opening, the rotary shaft and the opening control mechanism are controlled by the rotational speed control motor and opening control motor, respectively. The technical parameters of the fertilization system are presented in Table 1 .



1. Frame 2. Rotational speed control motor 3. Opening control motor 4. Rotary shaft 5. Opening control mechanism 6. Vertical fertilizer apparatus with spiral Geneva mechanism 7. Fertilizer bin 8. Fan 9. Pneumatic conveying pipes

Figure 1 Overall structure of the vertical fertilization system with spiral Geneva mechanism

Table 1 Technical parameters of the fertilization system

\begin{tabular}{lc}
\hline \multicolumn{1}{c}{ Parameter } & Value \\
\hline Control range of opening $/ \mathrm{mm}$ & $0-60$ \\
Control range of rotational speed $/ \mathrm{r} \cdot \mathrm{min}^{-1}$ & $5-20$ \\
Discharged amount of fertilizer $/ \mathrm{kg}$ & $10-100$ \\
Driving torque of single fertilizer apparatus $/ \mathrm{N} \cdot \mathrm{m}$ & 0.5 \\
Opening adjustment force of single fertilizer apparatus $/ \mathrm{N}$ & 15 \\
Fertilization mode & Strip fertilization
\end{tabular}

2.2 Working principle of vertical fertilization system with spiral Geneva mechanism

The key component of the vertical fertilization system with spiral Geneva mechanism is the vertical fertilizer apparatus with spiral Geneva mechanism. As shown in Figure 2, it is composed of the opening control mechanism, rotational speed control mechanism, spiral Geneva mechanism, opening baffle, and the shell of fertilizer apparatus.

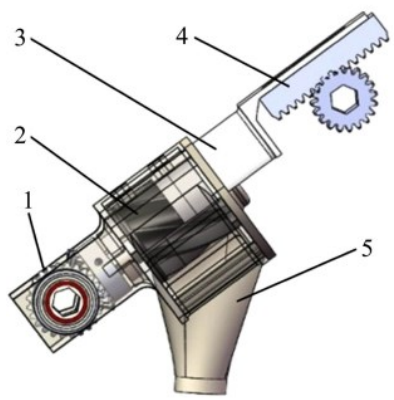

1. Rotational speed control mechanism 2. Spiral Geneva mechanism 3. Opening baffle 4. Opening control mechanism 5. Shell of fertilizer apparatus Figure 2 Overall structure of the vertical fertilizer apparatus with spiral Geneva mechanism

During operation, the opening control gear is driven by the opening control motor, and the gear rack mechanism drives the opening baffle, performing the real-time control of the opening size of the fertilization system. The rotational speed control motor is connected with the rotary shaft, which drives the fertilizer discharging wheel through a bevel gear transmission. The rotational speed is synchronized with the driving shaft to perform the real-time control of the rotational speed of the spiral Geneva mechanism.

During fertilization, once the fertilizer is discharged, it falls into the fertilizing ditch opened by the opener under the action of 
airflow. The fertilization process is completed by covering the fertilizer on the soil with a press wheel.

\section{EDEM based simulation and analysis of uniformity in fertilizer discharge}

\subsection{Fertilizer and fertilizer apparatus model}

The organic-inorganic compound fertilizer produced by Jibang company was taken as the modeling object in the simulation. It is found in the surveyed literature that the average triaxial dimensions (length, width, thickness) of the fertilizer are $3.31 \mathrm{~mm}, 3.23 \mathrm{~mm}$, $3.17 \mathrm{~mm}$, respectively, and its sphericity rate is $97.67 \%$. The equivalent diameter of the fertilizer is $3.24 \mathrm{~mm}$ after it is equivalent to a sphere ${ }^{[29]}$. Therefore, according to the average triaxial size, the spherical particle model with a radius of $1.62 \mathrm{~mm}$ is used for filling in order to shorten the simulation period. The particle model is shown in Figure 3.

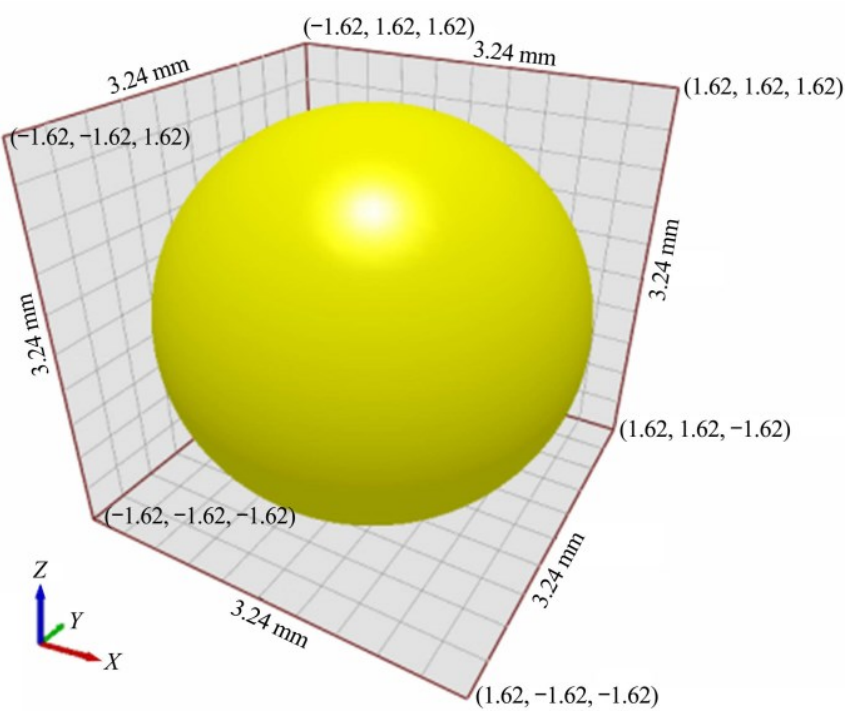

Figure 3 Schematic diagram of the fertilizer model

In order to analyze the discrete element simulation of the uniformity of the fertilizer apparatus, the whole machine with five lines of vertical spiral fertilizer apparatus was reduced to a single line, and the parts irrelevant to the fertilizer apparatus and the contact model were removed. The simulation model, developed by importing the $3 \mathrm{D}$ model into the EDEM software, is shown in Figure 4. The model is composed of a fertilizer box, fertilizer apparatus shell, fertilizer discharge wheel, generated geometry for fertilizer, etc. Since the contact collision between fertilizer particles, as well as between fertilizer and fertilizer distributor, is non-viscous and the time step is relatively small, the non-sliding Herz Mindlin model was used for measuring the contact between them.

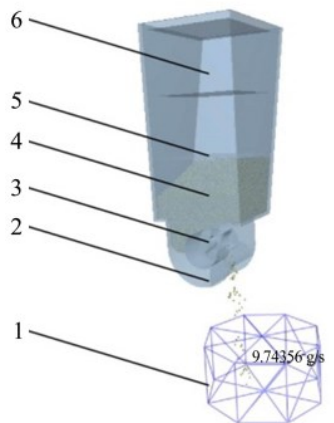

1. Mass flow rate sensor $\quad 2$. Shell of fertilizer apparatus 3 . Fertilizer discharge wheel 4. Fertilizer 5. Generated geometry of fertilizer 6. Fertilizer box

Figure 4 Schematic diagram of the simulation model
Considering that the setting of the global variables in the EDEM preprocessing has a great influence on the simulation, several pre-simulation calibration tests were carried out by taking the material calibration parameters of the two materials and the material interaction parameters between them from $\mathrm{Hu}$ et al. ${ }^{[30]}$ and Barrios et al. ${ }^{[31]}$ The parameters are listed in Table 2.

Table 2 Material properties and contact parameters

\begin{tabular}{cclc}
\hline \multirow{4}{*}{$\begin{array}{c}\text { Object } \\
\text { Material }\end{array}$} & \multicolumn{1}{c}{ Parameter } & Value \\
\cline { 2 - 4 } properties & pertilizer & Density $/ \mathrm{g} \cdot \mathrm{mm}^{-3}$ & 1.645 \\
& & Poisson's ratio & 0.25 \\
& Fertilizer & Shear modulus/Pa & $9.4 \times 10^{7}$ \\
\cline { 2 - 4 } & apparatus & Ponsity $/ \mathrm{g} \cdot \mathrm{mm}^{-3}$ & 7.810 \\
& & Shear modulus/Pa & 0.40 \\
& Contact between & Restitution coefficient of impact & 0.11 \\
fertilizers & Static friction coefficient & 0.30 \\
parameters & Rolling friction coefficient & 0.10 \\
\cline { 2 - 4 } & $\begin{array}{c}\text { Contact between } \\
\text { fertilizer and }\end{array}$ & Restitution coefficient of impact & 0.41 \\
& fertilizer apparatus & Static friction coefficient & 0.32 \\
& Rolling friction coefficient & 0.18 \\
\hline
\end{tabular}

3.2 Factors and principle analysis of simulation

In order to study the influence of different spiral angles of the fertilizer discharge wheel on the uniformity of fertilizer discharge, and to obtain better parameters for it, the EDEM simulation was carried out on the basis of the developed model of fertilizer particle and fertilizer discharge wheel. In the simulation, the parameters, such as the installation angle and opening, were kept constant, and a spiral Geneva mechanism with an effective working length of $30 \mathrm{~mm}$ was adopted. The spiral angle of the Geneva mechanism and the rotational speed of the fertilizer discharge wheel were selected as the simulation experimental factors, whose levels are shown in Table 3.

Table 3 Levels of the simulation experimental factors

\begin{tabular}{ccc}
\hline & \multicolumn{2}{c}{ Factor } \\
\cline { 2 - 3 } Level & Spiral angle $/\left({ }^{\circ}\right)$ & ${\text { Rotational speed } / \mathrm{r} \cdot \mathrm{min}^{-1}}^{2}$ \\
\hline 1 & 0 & 10 \\
2 & 15 & 20 \\
3 & 30 & 30 \\
4 & 45 & 40 \\
5 & 60 & 50 \\
\hline
\end{tabular}

The simulation model developed for the test is shown in Figure 4. The fertilizer particle model was generated by the fertilizer generation geometry 5 and it was put in the fertilizer box 6 . The contact force between particles was calculated at each time step. Then, according to Newton's second law and the relationship between force and displacement, the relationship among force, velocity, and displacement was established. In this study, a total of $1.5 \mathrm{~kg}$ of fertilizer particles are generated in $1 \mathrm{~s}$, and the fertilizer particles are accelerated to fall along the $z$-axis direction at the initial velocity of $-3 \mathrm{~m} / \mathrm{s}$ due to the gravity field. The fertilizer particles were driven out by the fertilizer wheel. Finally, those were measured by the mass flow sensor.

The simulation experiment was carried out for $14 \mathrm{~s}$. The output time interval of mass flow rate QMI was $0.1 \mathrm{~s}$, and a set of data consisting of 100 points were collected in 4-14 $\mathrm{s}$.

\subsection{Evaluation index}

3.3.1 Uniformity of fertilizer discharge

The fertilizer discharge should be uniform to meet the 
requirements of smooth fertilization. In order to accurately evaluate the effect of different spiral angles of the fertilizer discharge wheel on fertilizer uniformity in EDEM simulation experiment, the coefficient of variation of mass flow rate $(\mathrm{CV})$ can be used to evaluate the uniformity of fertilizer discharge and the stability of fertilizer discharge process, according to $\mathrm{JB} / \mathrm{T}$ 9783-2013 (Fertilizer apparatus with outer Geneva mechanism of the planter). The standard deviation of the mass flow rate $s$ of each data output node is calculated by using its coefficient of variation, which is used to judge the uniformity of fertilizer flow measured through the mass flow rate sensor area in a period of time. The mathematical formula is given in Equation (1).

$$
\begin{gathered}
\overline{Q_{m}}=\frac{1}{n} \sum Q_{m i} \\
s=\sqrt{\frac{1}{n-1} \sum\left(Q_{m i}-\overline{Q_{m}}\right)^{2}} \\
C V=\frac{s}{\overline{Q_{m}}} \times 100 \%
\end{gathered}
$$

where, $n$ is the total number of data output points; $Q_{m i}$ represents the mass flow rate at level $i, \mathrm{~g} / \mathrm{s} ; \overline{Q_{m}}$ indicates the average mass flow rate, $\mathrm{g} / \mathrm{s} ; s$ represents the standard deviation of the mass flow rate.

The coefficient of variation of mass flow rate (CV) is used as a parameter to evaluate the uniformity in the discharge of fertilizer. The smaller the CV, the better the uniformity is in the discharge of fertilizer. CV can be used to compare and analyze the performance of fertilizer apparatuses with different spiral angles.

\subsubsection{Linear correlation of fertilizer discharge}

The fertilizer discharge should be linear in correlation to meet the requirements of precision fertilization. In order to accurately evaluate the effect of different spiral angles of the fertilizer discharge wheel on the linear correlation of fertilizer discharge in the EDEM simulation experiment, the square of linear correlation coefficient $R_{\omega}^{2}$ of the regression equation is used as the evaluation index. The square of linear correlation coefficient $R_{\omega}^{2}$ is obtained by fitting the mean mass flow rate and the rotation speed of the fertilizer wheel. The mathematical formula is given in Equation (2).

$$
R_{\omega}^{2}=\left(\frac{\sum_{i=1}^{n}\left(\omega_{i}-\bar{\omega}\right)\left(Q_{m i}-\overline{Q_{m}}\right)}{\sqrt{\sum\left(\omega_{i}-\bar{\omega}\right)^{2}} \sqrt{\sum\left(Q_{m i}-\overline{Q_{m}}\right)^{2}}}\right)^{2}
$$

where, $\omega_{i}$ represents the rotational speed at level $i, \mathrm{rad} / \mathrm{s} ; \quad \bar{\omega}$ is the average rotational speed, $\mathrm{rad} / \mathrm{s}$.

The square of linear correlation coefficient $R_{\omega}^{2}$ is used as the parameter to evaluate the linear correlation of fertilizer discharge. A small value of $R_{\omega}^{2}$ indicates that the linear correlation between the mean mass flow rate and the rotational speed of the fertilizer wheel is not high, which will cause the linear decision-making to deviate from the actual fertilizer discharge amount, and affect the accurate discharge of fertilizer required in actual working conditions.

\subsection{Simulation results and analysis}

\subsubsection{Coefficient of variation of mass flow rate}

It can be seen in Figure 5 that the coefficient of variation of mass flow rate $(\mathrm{CV})$ decreases with increasing rotational speed. When the spiral angle is less than $60^{\circ}$, the CV first decreases and then increases with increasing the spiral angle. The minimum value of $\mathrm{CV}$ is obtained when the spiral angle is $45^{\circ}$. The uniformity and stability of fertilizer discharge are simultaneously affected by the rotational speed and spiral angle of the fertilizer discharge wheel.

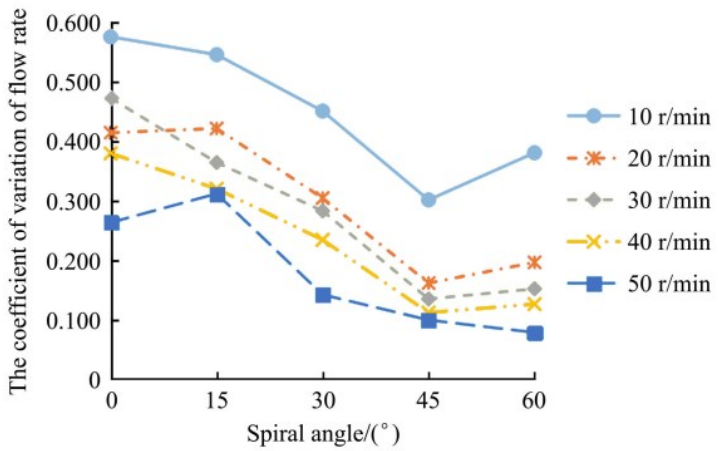

Figure 5 Line chart of rotational speed of fertilizer discharge wheel and variation coefficient of mass flow rate versus spiral angle

3.4.2 Effect of spiral angle on linear relationship between mass flow rate and rotational speed of fertilizer discharge wheel

It can be seen in Table 4 that $R_{\omega}^{2}$ reaches the maximum value at the spiral angle of $0^{\circ}$ and $45^{\circ}$, which indicates that the accuracy of the scheme using the rotational speed control strategy to adjust the mass flow rate is the best at those values of the spiral angle.

Combining the case of the minimum variation of mass flow rate $(\mathrm{CV})$ with the simulation experimental results presented in Table 4 , the spiral angle of $45^{\circ}$ is selected as the best parameter for the structural design of the spiral Geneva mechanism.

Table 4 Square of linear correlation coefficient of regression equation under different spiral angles

\begin{tabular}{cccccc}
\hline Spiral angle & $0^{\circ}$ & $15^{\circ}$ & $30^{\circ}$ & $45^{\circ}$ & $60^{\circ}$ \\
\hline$R_{\omega}^{2}$ & 0.9998 & 0.9336 & 0.9993 & 0.9998 & 0.9997 \\
\hline
\end{tabular}

\section{Experiment on static blowing fertilization}

\subsection{Experimental conditions}

The static blowing fertilization experiment was conducted in the indoor shed at the Zengcheng teaching and experimental base of the South China Agricultural University. The main experimental instruments included a contact velocimeter with a range of 0.5-19 999.0 r/min, high precision electronic balance with an accuracy of $0.01 \mathrm{~g}$, and an ordinary plastic bag. The experimental setup of the static blowing fertilization system is shown in Figure 6.

\subsection{Experimental indicators and methods}

In the static blowing fertilization experiment, the double factor test method was adopted at a certain wind speed, where the opening of the fertilizer discharging wheel and the rotational speed of the rotary shaft were taken as the test factors. The factors at different levels are shown in Table 5. Taking the average discharge of fertilizer, the consistency in discharge in each row and the coefficient of variation in the fertilizer stability as the test indicators, the linear relationships among the discharged amount of fertilizer, rotation speed, and opening of the fertilizer discharging wheel were studied. In addition to that, the relationships among the variation coefficient of the fertilizer discharging stability, rotation speed, and opening of the fertilizer discharging wheel were also studied. During the experiment, the fertilizer discharged in $10 \mathrm{~s}$ from each fertilizer discharged was collected in a plastic bag and weighed in the electronic balance. The process was repeated 3 times. The Equation for calculating the average amount of fertilizer discharge in the repeated test and the average amount of fertilizer discharge in four fertilizer apparatuses is given in Equation (3). 


$$
\bar{m}=\frac{1}{n} \sum m_{i}
$$

where, $\bar{m}$ denotes the average amount of fertilizer discharged, $\mathrm{g}$;

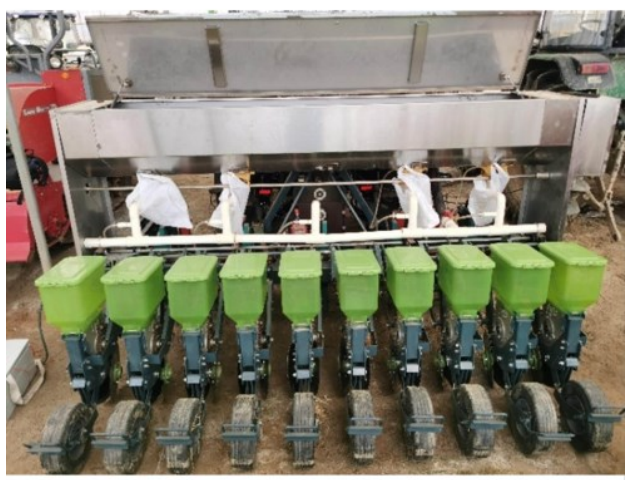

a. Physical drawing of the static test device $m_{i}$ is the amount of fertilizer discharged by a single fertilizer apparatus or a single group of fertilizer apparatuses, $g ; n$ represents the number of fertilizer apparatuses or the number of repetitions.



b. 3D model drawing of static test device

1. 2BDH-10 type precision rice hill-drop drilling machine for dry land 2. Vertical fertilizer apparatus with spiral Geneva mechanism

3. Fertilizer bin 4. Pneumatic conveying pipes

Figure 6 Experimental setup of the static air blowing fertilization system

Table 5 Factors and levels

\begin{tabular}{ccc}
\hline \multirow{2}{*}{ Level } & \multicolumn{2}{c}{ Factors } \\
\cline { 2 - 3 } & Opening/mm & Rotational speed $/ \mathrm{r} \cdot \mathrm{min}^{-1}$ \\
\hline 1 & 10 & 5 \\
2 & 20 & 10 \\
3 & 30 & 15 \\
4 & 40 & 20 \\
5 & 50 & -- \\
6 & 60 & -- \\
\hline
\end{tabular}

\subsection{Data for static experiment and result analysis}

4.3.1 Consistency in discharging amount in each row

The static experimental data are shown in Table 6 , and the coefficients of variation under different conditions are shown in Figure 7.

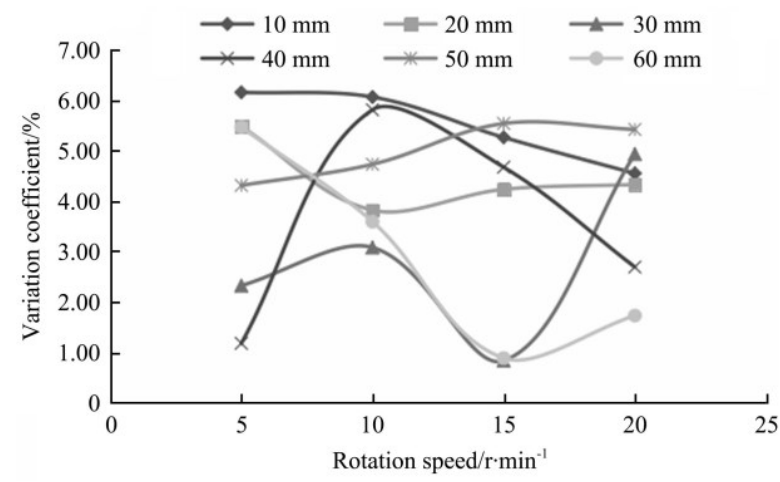

Figure 7 Coefficients of variation under different openings

It can be seen in Figure 7 that the maximum coefficient of variation is $6.16 \%$, which meets the requirement of fertilization.

Table 6 Fertilizer discharging performance of static air blowing

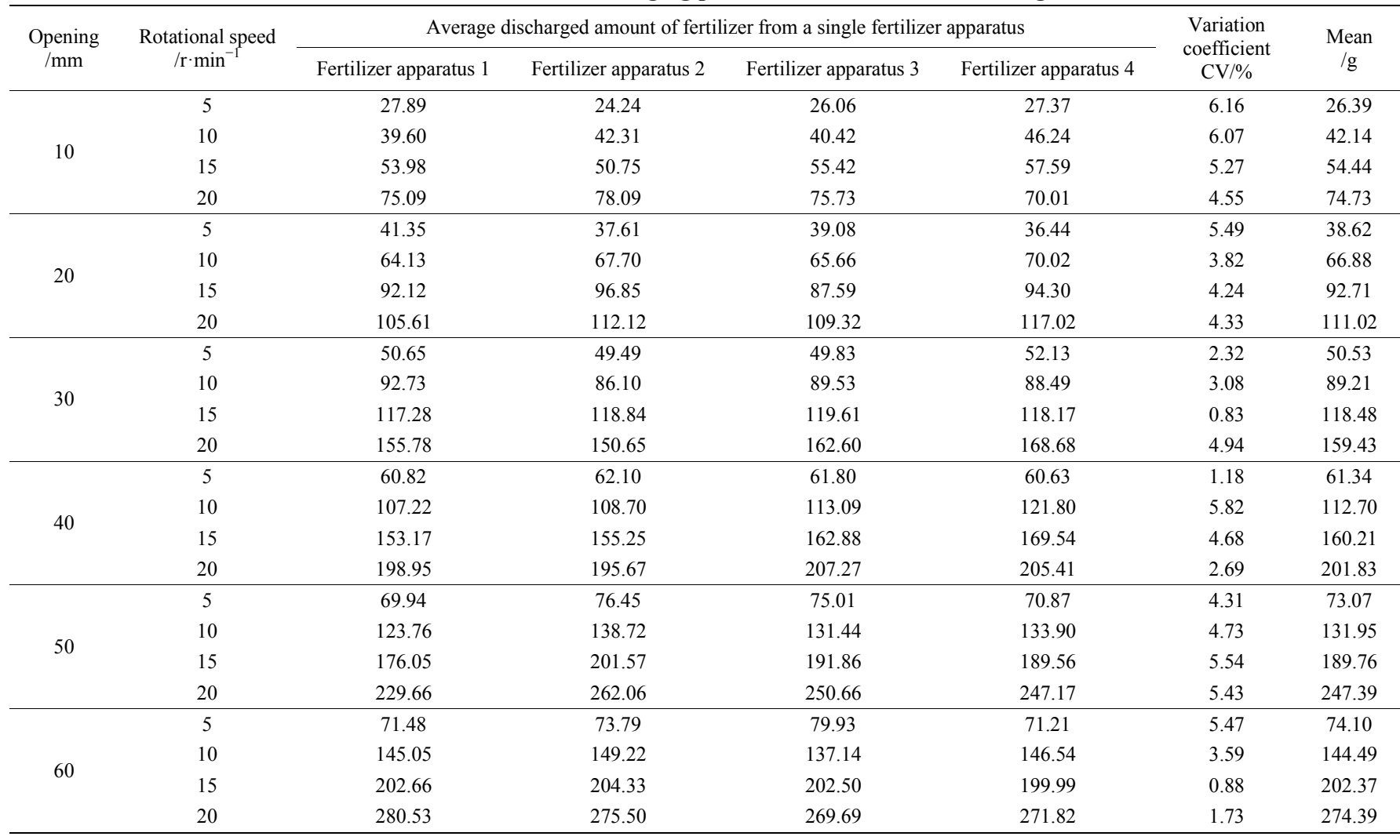


4.3.2 Effect of adjusting the opening of the fertilizer discharging wheel on the discharged amount of fertilizer

The average amount of fertilizer $\bar{m}$ discharged by the four fertilizer apparatuses under different conditions was calculated by Equation (3). A linear fitting method was used to develop the regression equations of the mean value $\bar{m}$ and the rotational speed of the fertilizer discharging wheel $\omega$, as well as the mean value $\bar{m}$ and the opening of the fertilizer wheel. Then, the correlation coefficients $R^{2}$ were calculated to study the linear relationships among the rotational speed, opening of the fertilizer discharging wheel and the discharged amount of fertilizer. The obtained results are presented in Tables 7 and 8 . The corresponding scatter diagrams are shown in Figures 8 and 9, respectively.

As shown in Table 7 and Figure 8, when the opening control strategy is prioritized, $R^{2}$ of the fitting curve shows an upward trend with the increasing opening. It indicates that the linear correlation between the discharged amount of fertilizer and the opening of the fertilizer discharging wheel is enhanced. The regression intercept of the fitting curve shows an upward trend with the increasing opening with a maximum value of 35.151 and a minimum value of 18.737, indicating decreasing precision performance of the fertilizer apparatus. In all the cases, $R^{2}$ values of the fitting curves fall in the range of 0.97-1 with a maximum value of 0.9953 . Therefore, the discharged amount of fertilizer can be adjusted linearly by adjusting the opening of the fertilizer discharging wheel.

\begin{tabular}{|c|c|c|}
\hline Opening/mm & Regression equation & $R^{2}$ \\
\hline 5 & $Q=1.0077 L+18.737$ & 0.9699 \\
\hline 10 & $Q=2.1084 L+23.85$ & 0.9891 \\
\hline 15 & $Q=3.0644 L+29.075$ & 0.9832 \\
\hline 20 & $Q=4.1423 L+35.151$ & 0.9953 \\
\hline
\end{tabular}

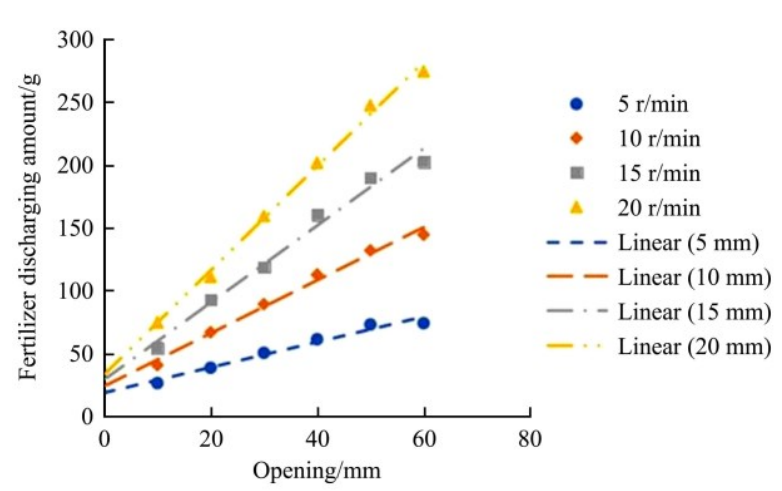

Figure 8 Scatter diagram and the variation of the regression equation of the discharged amount of fertilizer with the opening of the fertilizer discharging wheel

Figure 8 shows that the data points are located below the fitting curve when the opening is relatively small under different rotational speeds. The data points move gradually upward above the fitting curve, and then start to be under the fitting curve with a continuous increase in the opening. These indicate that the discharged amount of fertilizer has a positive correlation with the opening of the fertilizer discharging wheel under a constant rotation speed. However, the amount increases first, then decreases, and finally becomes stable. The reason for this phenomenon can be attributed to the uneven flow velocity of fertilizer in the spiral Geneva mechanism. The flow velocity near the inner wall is less than that in the middle area. However, the effect of the flow velocity on the fertilizer discharge decreases with an increase in the opening.

4.3.3 Effect of the rotational speed of the fertilizer discharging wheel on the discharged amount of fertilizer.

As shown in Table 8 and Figure 9, when the rotational speed control strategy is prioritized, $R^{2}$ of the fitting curve shows an upward trend with increasing rotational speed. It indicates that the linear correlation between the discharged amount of fertilizer and the rotational speed of the fertilizer discharging wheel is enhanced. The intercept of the regression equation of the fitting curve fluctuates slightly in the range of 9-16 with a maximum value of 16.775 and a minimum value of 9.15 , indicating that the precision of the fertilizer ejector is better than that of the opening priority control strategy. In all of the cases, $R^{2}$ values of the fitting curves fall in the range of $0.99-1$, which are larger than those of the opening priority control strategy. Therefore, the discharged amount of fertilizer can be adjusted linearly by adjusting the rotational speed of the fertilizer discharging wheel, whose accuracy is higher than that obtained by adjusting the opening of the wheel.

Table 8 Variation of the regression equation and $R^{2}$ of the discharged amount of fertilizer with the rotational speed of the fertilizer discharging wheel

\begin{tabular}{clc}
\hline Rotational speed $/ \mathrm{r} \cdot \min ^{-1}$ & Regression equation & $R^{2}$ \\
\hline 10 & $Q=3.1764 \omega+9.345$ & 0.9909 \\
20 & $Q=4.8606 \omega+16.55$ & 0.9913 \\
30 & $Q=7.1194 \omega+15.42$ & 0.9963 \\
40 & $Q=9.3796 \omega+16.775$ & 0.9978 \\
50 & $Q=11.615 \omega+15.35$ & 1 \\
60 & $Q=13.175 \omega+9.15$ & 0.9983 \\
\hline
\end{tabular}

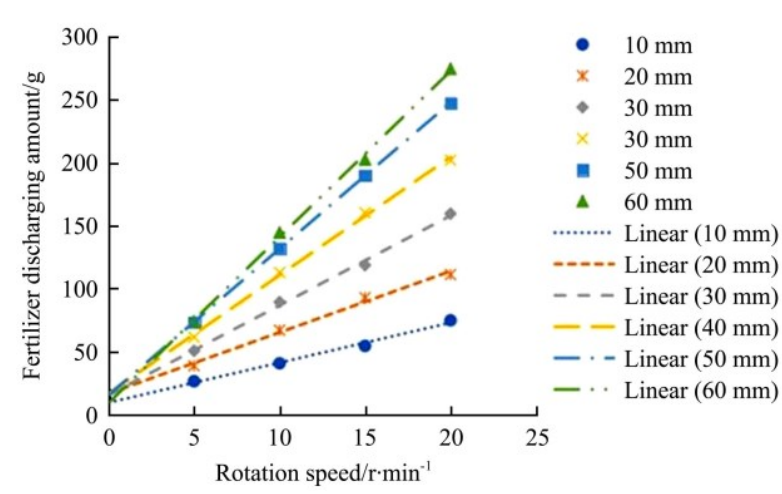

Figure 9 Scatter diagram and variation of regression equation of the discharged amount of fertilizer with a rotational speed of fertilizer discharging wheel

4.3.4 Variance analysis of static blowing experimental data

The multiple factor variance analysis of the static blowing experimental data was carried out in the Design Expert software. The obtained results are presented in Table 9. The results show that the $F$ value of the rotational speed is greater than that of the opening degree, but both the values are much greater than the rejection range of the $F$ ratio. In other words, under this condition, both rotational speed and opening have significant influence on the discharged amount of fertilizer, and the influence of the rotational speed is most significant. 
Table 9 Variance analysis of the mean value of the discharged amount of fertilizer

\begin{tabular}{cccccc}
\hline Source of variance & Square sum of variance $S$ & DOF $f$ & Average variance $S / f$ & $F$ ratio & Significance marker \\
\hline Rotation speed & 152134.8 & 3 & 50711.6 & 2599.9 & $F_{\text {a }}$ \\
Opening & 141200.5 & 5 & 28240.1 & 1447.8 & $* *$ \\
Error & 936.2 & 48 & 19.5 & -- & -- \\
Sum & 293335.3 & 56 & -- & $F_{0.1(5,48)}=1.97$ & -- \\
\hline
\end{tabular}

\subsubsection{Regression model of variation coefficient}

The mean values of the variation coefficients of the four fertilizer discharged were taken as the test indicators. The variance analysis of the static blowing experimental results was conducted in the Design Expert software. The obtained results show that the rotational speed and the opening of the fertilizer discharging wheel have significant effect on the experimental indicator. The regression equation of the mean value of the variation coefficients of the fertilization stability, affected by the rotational speed and the opening of the fertilizer discharging wheel opening, was obtained by curve fitting given by Equation (4).

$$
\begin{aligned}
y_{1}= & 23.78625-2.02182 \omega-0.34725 L+0.010032 \omega L+ \\
& 0.052767 \omega^{2}+0.002729 L^{2}
\end{aligned}
$$

Equation (4) indicates that the average coefficient of variation $y_{1}$ of the discharged amount of fertilizer decreases with increasing rotational speed and opening within certain ranges. However, the coefficient of variation $y_{1}$ increases in a small range with the increasing rotational speed or opening.

According to "NY/T1003-2006 technical specification for

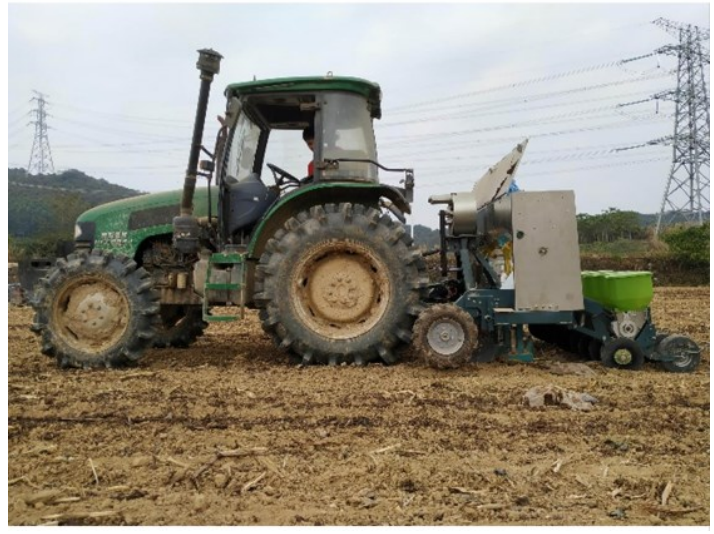

a. Physical drawing of the device in the field test

Note: 1-4 indicate fertilizer apparatus 1-4, respectively.

Figure 10 Field experiment on fertilization

\subsection{Experimental conditions}

The length and width of the selected field were $61.0 \mathrm{~m}$ and $47.8 \mathrm{~m}$, respectively. The soil type was loam soil with a moisture content of $18 \%-25 \%$. Ahead of the experiment, rotary tillage was carried out, and the surface was smoothened and loosened. The experimental materials and instruments were the same as those used in the static blowing fertilization experiment.

\subsection{Experimental methods}

In the field experiment, the same experimental factors and levels were used as in the static blowing fertilization experiment. The variation coefficient of the fertilizer discharging stability at each fertilizer discharged was taken as a test indicator. The driving crew drove in the field at a speed of about $2.5 \mathrm{~km} / \mathrm{h}$ to verify the relationship between the variation coefficient of the fertilizer discharging stability at each fertilizer discharged and the opening. During the experiment, the fertilizer discharged in $10 \mathrm{~s}$ from each fertilizer discharged was collected in a plastic bag, and weighed in the electronic balance. The process was repeated quality evaluation of fertilization machinery" issued by the Ministry of Agriculture in 2006 and the regression equation of the coefficient of variation, the Equation was solved in MATLAB $\mathrm{R} 2014 \mathrm{~b}$ with constraint $\left(y_{1}<7.8 \%\right)$. The obtained results show that $y_{1}<7.8 \%$ can be achieved when the opening of the fertilizer discharging wheel is $15.46-49.40 \mathrm{~mm}$ and its rotational speed is $7.73-24.7 \mathrm{r} / \mathrm{min}$. When the rotation speed is $15.9 \mathrm{r} / \mathrm{min}$ and the opening is $34.4 \mathrm{~mm}, y_{1}$ achieves the minimum value of $1.750 \%$.

\section{Experiment on-field performance verification}

\subsection{Purpose of the experiment}

In order to verify the working performance of the vertical spiral fertilizer apparatus, field experiments were carried out in June 2019 and January 2020 in the experimental field of the Zengcheng teaching and research base of the South China Agricultural University. The experiments were carried out with a 2BDH-10 type precision rice hill-drop drilling machine for dry land and vertical spiral fertilization system. The field experiment is shown in Figure 10.

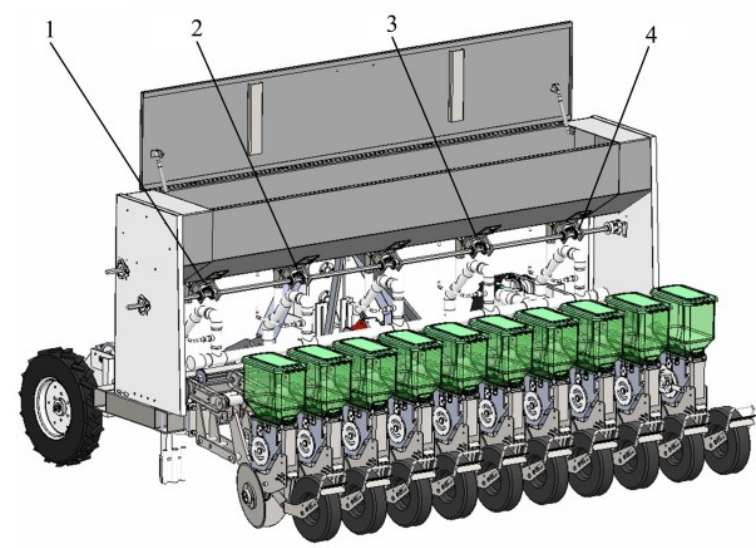

b. 3D model drawing of the device in the field test
3 times.

\subsection{Field experimental data and result analysis}

The field dynamic air blowing experimental data are shown in Table 10, and the variation coefficient of each fertilizer discharged with an opening is shown in Figure 11. Table 10 shows that the average variation coefficient $y_{1}$ of the amount of fertilizer discharged from the field air blowing experiment has a little deviation from the theoretical value obtained by Equation (2) under different conditions. However, the changing trend is similar to the theoretical one, both decreasing with an increase in the opening. The minimum value is obtained when the opening is $40-50 \mathrm{~mm}$, and then it increases in a small range with an increase in the opening.

Figure 11 shows that the variation coefficient of each fertilizer discharged decreases first and then increases with an increase in the opening at different rotational speeds. The minimum value occurred when the opening was $40 \mathrm{~mm}$ or $50 \mathrm{~mm}$, which is consistent with the changing trend of the theoretical value.

The obtained results show that if the opening control strategy 
is prioritized at the opening value of $40 \mathrm{~mm}$ or $50 \mathrm{~mm}$, the variation coefficient of stability of each fertilizer apparatus reaches the minimum value, which is much smaller than the qualified index of $7.8 \%$.

Table 10 Variation coefficient of stability of each fertilizer apparatus

\begin{tabular}{|c|c|c|c|c|c|c|c|}
\hline \multirow{2}{*}{$\begin{array}{l}\text { Rotational speed } \\
\qquad / \mathrm{r} \cdot \mathrm{min}^{-1}\end{array}$} & \multirow{2}{*}{$\begin{array}{l}\text { Opening } \\
/ \mathrm{mm}\end{array}$} & \multicolumn{4}{|c|}{ Variation coefficient of stability of each fertilizer apparatus $/ \%$} & \multirow{2}{*}{ Mean value $/ \%$} & \multirow{2}{*}{ Theoretical value $/ \%$} \\
\hline & & 1 & 2 & 3 & 4 & & \\
\hline \multirow{6}{*}{5} & 10 & 1.15 & 4.75 & 4.32 & 9.50 & 4.93 & 12.30 \\
\hline & 20 & 11.97 & 1.21 & 6.63 & 4.63 & 6.11 & 10.15 \\
\hline & 30 & 10.04 & 4.31 & 4.27 & 2.32 & 5.24 & 8.54 \\
\hline & 40 & 6.80 & 2.67 & 3.31 & 1.49 & 3.57 & 7.48 \\
\hline & 50 & 3.02 & 8.75 & 5.87 & 2.64 & 5.07 & 6.96 \\
\hline & 60 & 12.38 & 11.50 & 1.59 & 6.91 & 8.10 & 7.00 \\
\hline \multirow{6}{*}{10} & 10 & 6.54 & 11.01 & 6.15 & 7.64 & 7.83 & 6.65 \\
\hline & 20 & 4.50 & 5.92 & 1.20 & 2.35 & 3.49 & 5.00 \\
\hline & 30 & 6.59 & 8.56 & 4.51 & 6.67 & 6.58 & 3.89 \\
\hline & 40 & 5.01 & 2.98 & 4.60 & 5.29 & 4.47 & 3.33 \\
\hline & 50 & 0.53 & 2.45 & 1.40 & 1.86 & 1.56 & 3.32 \\
\hline & 60 & 1.82 & 2.77 & 4.57 & 1.50 & 2.66 & 3.85 \\
\hline \multirow{6}{*}{15} & 10 & 14.78 & 12.12 & 7.72 & 2.47 & 9.27 & 3.64 \\
\hline & 20 & 9.31 & 8.70 & 3.14 & 5.90 & 6.76 & 2.49 \\
\hline & 30 & 4.32 & 2.76 & 4.23 & 5.89 & 4.30 & 1.88 \\
\hline & 40 & 2.09 & 2.54 & 4.86 & 1.49 & 2.75 & 1.83 \\
\hline & 50 & 8.59 & 1.87 & 5.91 & 2.83 & 4.80 & 2.32 \\
\hline & 60 & 2.20 & 5.67 & 8.44 & 1.90 & 4.55 & 3.35 \\
\hline \multirow{6}{*}{20} & 10 & 11.28 & 6.34 & 5.19 & 1.41 & 6.05 & 3.26 \\
\hline & 20 & 4.70 & 2.59 & 5.31 & 2.85 & 3.86 & 2.62 \\
\hline & 30 & 0.90 & 1.25 & 1.68 & 4.01 & 1.96 & 2.51 \\
\hline & 40 & 0.79 & 2.45 & 0.53 & 2.18 & 1.49 & 2.96 \\
\hline & 50 & 8.59 & 3.51 & 8.21 & 3.55 & 5.97 & 3.95 \\
\hline & 60 & 2.39 & 5.34 & 6.23 & 1.46 & 3.86 & 5.48 \\
\hline
\end{tabular}

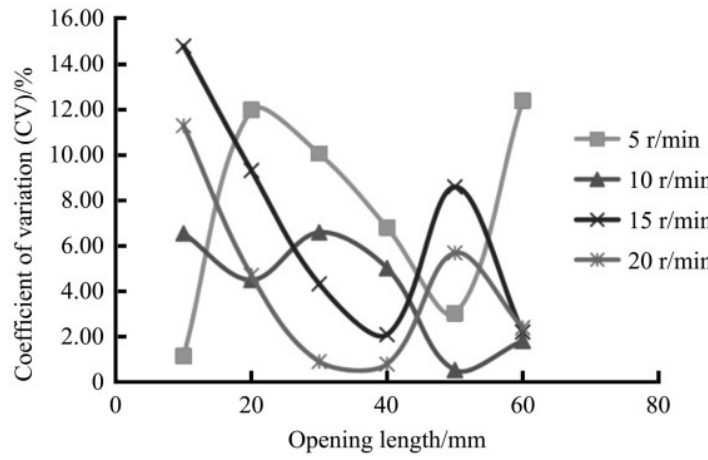

a. Fertilizer apparatus1

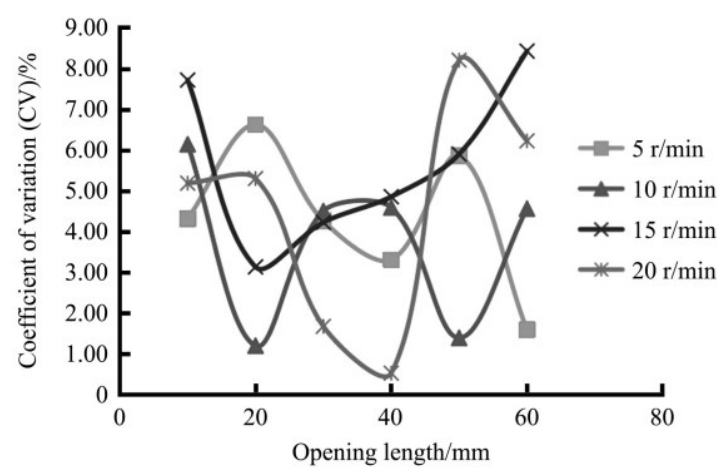

c. Fertilizer apparatus 3

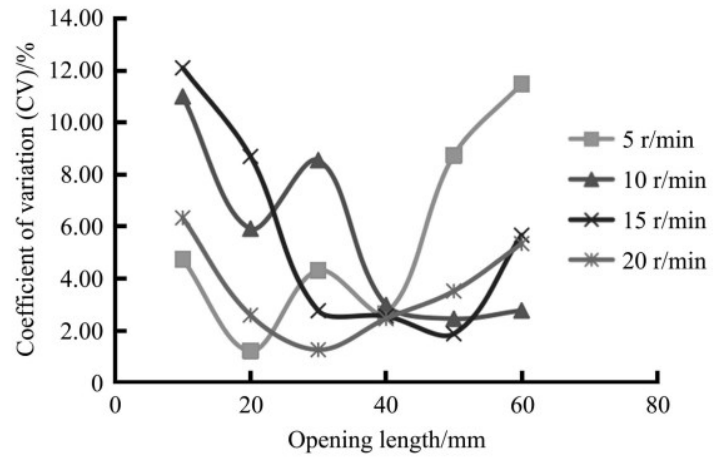

b. Fertilizer apparatus 2

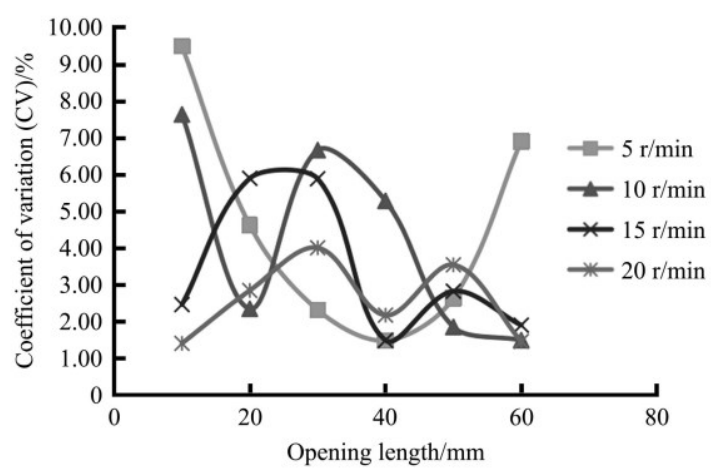

d. Fertilizer apparatus 4

Figure 11 Variation coefficient of each fertilizer apparatus with opening

\subsection{Difference analysis of test results}

According to the regression equation of the coefficient of variation fitted by a static blowing test, the optimum opening of the fertilizer discharge baffle was $34.4 \mathrm{~mm}$, which was inconsistent 
with the field test result of $40-50 \mathrm{~mm}$. Its main reason is that when the air blowing test was carried out in the field, the variation in the coefficient of the fertilizer discharge stability of the fertilizer apparatus with small and medium opening was larger than that of the static blowing test due to the vibration caused by the running fan, machine walking and fluidity of fertilizer particles.

\section{Discussion}

When a vertical pneumatic fertilization system with a spiral Geneva mechanism having a helix angle of $45^{\circ}$ was tested, a good linear correlation between the amount of fertilizer discharge and the opening and rotational speed, and the fertilizer discharge was obtained. Therefore, accurate control of the fertilizer discharge can be realized by adjusting the rotational speed or opening of the fertilizer discharge wheel. The linear correlation and fertilizer discharge accuracy under control of the rational speed are better than those under the opening control. The effect of rotational speed on the fertilizer discharge and average coefficient of variation is more significant than that of opening. With the limitations in experimental conditions and testing equipment, the comprehensive influence of rotational speed and the opening degree of the fertilizer wheel on the fertilizer discharge accuracy and their relationship, and the influence of various factors on the discharged amount of fertilizer with different opening degrees in field operation need to be studied further.

\section{Conclusions}

1) In this study, a kind of vertical pneumatic fertilization system with a spiral Geneva mechanism was designed. The rotary shaft and the opening control mechanism were controlled by a rotational speed control motor and an opening control motor, respectively, to perform bi-variable control of the rotational speed and the opening of the fertilizer discharging wheel, thus achieving the purpose of precise fertilization. The fertilizer was transported by air flow in order to avoid any blockage during the discharge of fertilizer.

2) The simulation results show that the accuracy of the scheme using the rotational speed control strategy to adjust the mass flow rate is the best at the spiral angle of $0^{\circ}$ and $45^{\circ}$. When the spiral angle was less than $60^{\circ}$, the coefficient of variation of mass flow rate $(\mathrm{CV})$ first decreases and then increases with increasing spiral angle, and its minimum value was obtained when the spiral angle was $45^{\circ}$. Therefore, the optimum spiral angle of the fertilizer discharge wheel was selected to be $45^{\circ}$.

3) The static experimental results show that the discharged amount of fertilizer has a good linear correlation and accuracy with the opening and rotational speed, whose best values are found with the rotational speed. The rotational speed and opening have significant effects on the discharged amount of fertilizer and the average coefficient of variation. The effect of the rotational speed was found most significant. According to the model obtained by multiple regression fittings, the optimal parameters are determined when the average coefficient of variation is small, the rotational speed is $15.9 \mathrm{r} / \mathrm{min}$ and the opening is $34.4 \mathrm{~mm}$.

4) The field experiment shows that the variation coefficient of each fertilizer discharged decreases first and then increases with an increasing opening under different rotational speeds, which is consistent with the changing trend of the theoretical value. Additionally, when the opening was $40 \mathrm{~mm}$ or $50 \mathrm{~mm}$, the variation coefficient reaches the minimum value, which was much smaller than the qualified index of $7.8 \%$.

\section{Acknowledgements}

The authors gratefully acknowledge the support was provided by the National Key Research and Development Program of China (Grant No. 2017YFD0700700; 2017YFD0700704; 2016YFD0200600; 2016YFD0200606; 2018YFD0200700).

\section{[References]}

[1] Ministry of Agriculture and Rural Affaires of the People's Republic of China. Ten-tear development plan of Mechanization of rice production in China (2006-1015): Available: http://www.moa.gov.cn/gk/ghjh_1/201006/ t20100606_1534739.htm. Accessed on [2020-01-09]

[2] Liu B Y. Control method of variable rate fertilization research. Harbin: Northeast Forestry University, 2013; 6. 267p. (in Chinese)

[3] Yuan W S, Jin M, Wu C Y, Xiao T Q. Development status and prospect of seed manure fertilizing mechanization in China. Journal of Agricultural Mechanization Research, 2011; 12: 1-5. (in Chinese)

[4] Gao Q, Liu S X, Wang Y, Chen L R. Effects of fertilization on agricultural ecological environment. Journal of Jilin Agricultural University, 2000; 22(Album): 106-112. (in Chinese)

[5] Wang H Y, Zhou J M. Calculation of real fertilizer use efficiency and discussion on fertilization strategies. Acta Pedologica Sinica, 2014; 51(2) 216-225.

[6] Yang Q L, Sang L M, Sun J R, Ji Z Q, Yuan W L, Guo Y W, et al. Current situation of fertilizer use in China and the method to improve chemical fertilizer utilization efficiency. Journal of Shanxi Agricultural Sciences, 2011; 39(7): 690-692.

[7] Wang H Y, Li J. Improve the application technology of chemical fertilizers. Agriculture and Technology, 2014; 34(7): 104. (in Chinese)

[8] Koch B, Khosla R, Frasier W M, Westfall D G, Inman D. Economic feasibility of variable-rate nitrogen application utilizing site-specific management zones. Agron. J, 2004; 96: 1572. doi: 10.2134/agronj2004.1572.

[9] Qi J T, Tian X L, Li Y, Fan X H, Yuan H F, Zhao J L, et al. Design and experiment of a subsoiling variable rate fertilization machine. Int J Agric \& Biol Eng, 2020; 13(4): 118-124.

[10] Zha X T, Zhang G Z, Zhang S J, Hou Q X, Wang Y, Zhou Y. Design and experiment of centralized pneumatic deep precision fertilization device for rice transplanter. Int J Agric \& Biol Eng, 2020; 13(6): 109-117.

[11] Chen M, Yang Z J, Wang X C, Shi Y Y, Zhang Y. Response characteristics and efficiency of variable rate fertilization based on spectral reflectance. Int J Agric \& Biol Eng, 2018; 11(6): 152-158.

[12] Du R C, Gong B C, Liu N N, Wang C C, Yang Z D, Ma M J. A design and experiment on intelligent fuzzy monitoring system for corn planters. Int J Agric \& Biol Eng, 2013; 6(3): 11-18.

[13] Zhou W Q, Wang J W, Tang H. Structure optimization of cam executive component and analysis of precisely applying deep-fertilization liquid fertilizer. Int J Agric \& Biol Eng, 2019; 12(4): 104-109.

[14] Yang L, Chen L, Zhang J, Liu H, Sun Z, Sun H, et al. Fertilizer sowing simulation of a variable-rate fertilizer apparatus based on EDEM. IFAC-PapersOnLine, 2018; 51: 418-423.

[15] Shi Y Y, Hu Z Z, Wang X C, Odhiambo M O, Sun G X. Fertilization strategy and application model using a centrifugal variable-rate fertilizer spreader. Int J Agric \& Biol Eng, 2018; 11(6): 41-48.

[16] Chen C, He P, Zhang J, Li X, Ren Z, Zhao J, et al. A fixed-amount and variable-rate fertilizer apparatus based on pulse width modulation. Comput. Electron. Agr, 2018; 148: 330-336.

[17] Zeng S, Tan Y P, Wang Y, Luo X W, Yao L M, Huang D P, et al. Structural design and parameter determination for fluted-roller fertilizer applicator. Int J Agric \& Biol Eng, 2020; 13(2): 101-110.

[18] Su N, Xu T S, Song L T, Wang R J, Wei Y Y. Variable rate fertilization system with adjustable active feed-roll length. Int $\mathrm{J}$ Agric \& Biol Eng, 2015; 8(4): 19-26.

[19] Chen X F, Luo X W, Wang Z M, Zhang M H, Hu L, Yang W W, et al. Design and experiment of fertilizer distribution apparatus with double-level screws. Transactions of the CSAE, 2015; 31(3): 10-16. (in Chinese)

[20] Pan S Q, Zhao Y X, Jin L, Qu G B, Tian Y. Design and experimental research of external grooved wheel fertilizer apparatus of 2BFJ-6 type variable rate fertilizer application. Journal of Chinese Agricultural Mechanization, 2016; 37(1): 40-42. (in Chinese)

[21] Shi Y Y, Chen M, Wang X C, Odhiambo M O, Zhang Y N, Ding W M. Analysis and experiment of fertilizing performance for precision fertilizer apparatus in rice and wheat fields. Transaction of the CSAM, 2017; 48(7): 


\section{7-103. (in Chinese)}

[22] Yuan J, Liu C, Li Y, Zeng Q, Zha X F. Gaussian processes based bivariate control parameters optimization of variable-rate granular fertilizer apparatus. Comput. Electron. Agr, 2010; 70: 33-41.

[23] Lv H, Yu J Q, Fu H. Simulation of the operation of a fertilizer spreader based on an outer groove wheel using a discrete element method. Mathematical and Computer Modelling, 2013; 58: 842-851.

[24] Ishola T A, Yahya A, Shariff A R M, Aziz S A. Novel variable rate pneumatic fertilizer applicator. Instrumentation Science \& Technology, 2014; 42(4): 369-384.

[25] Zhang T, Liu F, Liu Y Q, Zhao M Q, Zhang S, Li N, et al. Discrete element simulation of outer groove wheel type fertilizer discharging device capacity analysis. Journal of Agricultural Mechanization Research, 2015; 37(09): 198-201.

[26] Ding S, Bai L, Yao Y, Yue B, Fu Z, Zheng Z, et al. Discrete element modelling (DEM) of fertilizer dual-banding with adjustable rates. Comput. Electron. Agr., 2018; 152: 32-39.
[27] Li K, Zhang L X, Zhang L P, Chu S Z, Qi X M, Qian Y, et al. Design and experiment of bivariate fertilizer. Journal of Gansu Agricultural University, 2016; 51(4): 128-133. (in Chinese)

[28] Wang Z M, Luo X W, Tang X R, Ma G H, Zhang G Z, Zeng S. Precision rice hill-direct-seeding technology and machine based on the combination of agricultural machinery and agronomic technology. Journal of South China Agricultural University, 2010; 31(1): 91-95. (in Chinese)

[29] Yang Z, Zhu Q C, Sun J F, Chen Z C, Zhang Z W. Study on the performance of fluted roller fertilizer distributor based on EDEM and 3D Printing. Journal of Agricultural Mechanization Research, 2018; 40(5): 175-180. (in Chinese)

[30] Hu G M. Analysis and simulation of granular system by discrete element method using EDEM. Wuhan: Wuhan University of Technology Press, 2010. (in Chinese)

[31] Barrios G K P, Carvalho R M, Kwade A, Tavares L M. Contact parameter estimation for DEM simulation of iron ore pellet handling. Powder Technology, 2013; 248: 84-93. 